\title{
Enhanced Numeral Recognition for Handwritten Multi-language Numerals Using Fuzzy Set-Based Decision Mechanism
}

\author{
Ahmad Al-Hmouz, Ghazanfar Latif, Jaafar Alghazo, and Rami Al-Hmouz
}

\begin{abstract}
Handwritten character and numeral recognition have gained interest in the research community as part of the big picture of Machine Learning. Writer independent recognition systems are still in the working and the research is geared towards an optimized technique that can achieve this. In this paper, we propose a numeral recognition system that forms fuzzy sets of the features extracted using modified structural features for English, Arabic, Persian, and Devanagari Numerals. The structural features extract the geometrical primitives that distinguish each image. After the feature extraction phase, the results are input into a classifier, we test two different classifiers namely Neural Network and Naïve Base. To further enhance the recognition process with low overhead the erroneously recognized numerals (confusion matrix) are processed through the fuzzy set-based decision mechanism to enhance the numeral recognition process. Results indicate that recognition is enhanced by applying the fuzzy set-based decision mechanism for both classifer.
\end{abstract}

Index Terms-Fuzzy decision, trapezoidal fuzzy sets, multi-language numerals, structural features.

\section{INTRODUCTION}

Online and offline character and numeral recognition is a topic of significant importance in the field of image recognition. The applications for online and offline recognition varies with the end product use. The difference between the two is well known; online dealing with spatio-temporal representation while offline dealing with spatio-luminance representation. Though online is a bit more challenging than offline recognition yet most proposed systems in previous literature are tested on offline datasets even if the system is proposed for an online setting. Dealing with Arabic and Persian digits in particular is different in some sense due to the absence of a comprehensive benchmark for these languages [1].

In this paper, we present an enhanced English, Arabic, Persian, and Devanagari numerals recognition system based on structural feature extraction, fuzzy set-based decision mechanism (Trapezoidal Fuzzy sets). The proposed system is put through a rigorous test on more than 190,000 handwritten numerals that exist in four different benchmark datasets. The output scores of the classifiers are quantified using fuzzy

Manuscript received May 23, 2019; revised December 15, 2019.

Ahmad Al-Hmouz is with the Faculty of Information Technology, Middle East University, Amman, Jordan (e-mail: aa998@uowmail.edu.au).

Ghazanfar Latif and Jaafar Alghazo are with the College of Computer Engineering and Sciences, Prince Mohammad bin Fahd University, Khobar, Saudi Arabia.

Rami Al-Hmouz is with the Department of Electrical and Computer Engineering, King Abdulaziz University, Jeddah, Saudi Arabia. membership functions. The parameters of the membership functions are optimized using particle swarm optimization. Results indicate that the use of fuzzy logic after classification results in better recognition rate and reduces the percentage of numbers erroneously recognized.

The organization of the rest of the paper is as follows: Section II highlights relevant literature, Section III details the methodology used for Numeral Recognition and details the fuzzy set-base decision mechanism (Trapezoidal Fuzzy sets) used to enhance the recognition process. Section IV lists and describes the datasets used in this paper, Section V details the results obtained prior to and after using fuzzy set-based decision mechanism, Section VI concludes the paper and set the direction for future work.

\section{LITERATURE REVIEW}

Numeral recognition algorithms have been proposed in various published research. To date an optimized numeral recognition algorithm is still not found. Previous work has concentrated on both online and offline digit recognition, however, in most cases even those algorithms proposed for online recognition are tested on offline datasets. In [2], the authors proposed a generic recognition algorithm for Arabic digits with the three main phases of Pre-processing, Feature extraction and classification. Their method was tested a small dataset consisting of 100 samples from 100 users.

Due to the size of the dataset, a recognition rate of $98 \%$ was reported. In [3], Bayesian fusion process was applied on certain parts of the confused characters in a way to improve the classification rate, the obtained classification rate was 97.95\%. In [4], the authors proposed a technique for Arabic digit recognition separating the zero digit from the digits 1-9. The algorithm utilizing both the spatial and temporal information achieves an average of $98.73 \%$ recognition rate. The contributions of the author also includes the introduction of a training set of 30,000 samples. In [5], Feature extraction using global features was proposed. These include global features extracted from Discrete Cosine Transform (DCT), Discrete Fourier Transform (DFT) as well as Wavelet Transform (WT).

Local features were extracted from other sources such as the nth derivative, two-dimensional coordinates, angular features and curvature. Experimental tests were done on global features, local features and combined features. An average of more than $95 \%$ recognition accuracy was achieved using the combined features. In [6], Persian characters were the target for a recognition algorithm that divided the letters into 18 groups based on structure and 
shape of the body of the character. The authors proposed the use of Hidden Markov Model (HMM) for the recognition of main body variables and delayed strokes with their HMM was used for final recognition. The proposed method achieved an accuracy recognition rate of $94.2 \%-95.9 \%$ for different groups. In [7], a comparative study was done between Multilayer Perceptron (MLP) and K-Nearest Neighbors (KNN) for the recognition of handwritten cursive Arabic numerals. Feature extraction utilized the morphological mathematical model. In this study, MLP produced better recognition rates compare with the KNN. Another comparative study was done in [8] between SVM and HMM on the recognition of Arabic numerals. SVM prevailed in this study over HMM in the recognition of Arabic numerals.

Persian-Arabic numeral recognition algorithm proposed in [9] based on robust feature set and the use of KNN classification. Contour form of each digit was obtained and three features were extracted using block base methods. The method achieved a recognition accuracy between 99.82\% $99.90 \%$. In [10], a new method was proposed for Persian-Arabic handwritten digit recognition. Feature set in the feature extraction phase was done by combining four Histogram of Oriented Gradient (HOG) and directional Chain Code Histogram (CCH).

Feature were extracted at two level yielding a higher recognition rate. 164 dimension were presented in their feature set. Radial basis function kernel SVM was utilized during the classification and the average recognition rate of 99.31\% was achieved on test datasets. In [11], Convolutional Neural Networks (CNN) was proposed for Arabic numeral recognition in addition to introducing a new dataset with 45,000 samples. This produced an average recognition rate of 95.7\%. 115 features for the recognition of Persian handwritten numeral was proposed in [12]. Ensemble classifier was used in the classification phase achieving an average recognition rate of $95.28 \%$. In [13], the authors proposed a feature set calculated from the vertical and horizontal directions of the image along with freeman chain code histogram $(\mathrm{CCH})$.

SVM was used in the classification phase. The results indicated that the recognition results were enhanced using this method. Deep Learning Algorithms have also been proposed for the recognition of Arabic and Persian numerals. In [14], a Convolutional Neural Network (CNN) was designed and called LeNet-5 for the recognition of handwritten Arabic digits. MADBase dataset was used for testing and results showed significant improvement over different classification algorithms. In [15], the authors developed a $\mathrm{CNN}$ for the recognition of mutli-language numerals in the following languages (English, Arabic, Persian, Urdu, and Devanagari). The overall accuracy of the combined dataset was $99.26 \%$ with a precision of $99.29 \%$.

Fuzzy and fuzzy based systems were also proposed for the recognition of numerals. In [16], the authors proposed a fuzzy based technique for the recognition of Arabic numerals. Directional features were used for automatically generation the fuzzy models in addition to automatic weight generation for the different segments. Automatic estimation of the fuzzy intervals using the training dataset was also included in their proposed algorithm. Then classification was done on two phases; first phase zero/nonzero classifications using features of the numerals and SVM as classifier, second phase classification of number 1-9 using the fuzzy classification using segment directional and histogram features and syntactic fuzzy classified. The first phase of zero/nonzero produced an average accuracy of $99.55 \%$ and second phase numbers 1-9 produced an average accuracy of $98.01 \%$.

In Ref. [17], the authors present a technique for handwritten Hindi and English digit recognition using their exponential membership functions as a base for a fuzzy model. The recognition process consists of modifying the membership function fitted to the fuzzy set which are derived from the features calculated from the normalized distances using the box approach. The overall average recognition accuracy was found to be $98.4 \%$ for English digits and $95 \%$ for Hindi digits. In [18], the authors propose a digit recognition method based on a simplified structural classification through the use of small set of primitive types along with fuzzy memberships. The algorithm extracts five types of primitive segments of each image based on three types of feature points.

An estimate of the likelihood of these primitives being close to the vertical boundaries of the image is calculated using the membership function. The classifier in their algorithm uses the primitives, extracted feature points and fuzzy memberships to classify the digits. Using the NIST dataset for testing, they achieved a recognition rate of $87.33 \%-88.72 \%$. In [19], another algorithm is proposed that forms the fuzzy sets from the extracted features.

The modified exponential membership function of type-2 is used to represent the input fuzzy sets. Fuzzy measure theory is used to manage the interaction between the input fuzzy sets. The algorithm was tested on the recognition of English and Devanagari digits as well as English characters. In all cases, the algorithm proved to achieve improved recognition over other methods. In [20], the recognition of Hindi handwritten digit recognition is the target of the study. This algorithm is also based on the modified exponential membership function that is fitted to fuzzy sets. This is derived from the extracted features containing the normalized distances based on the box approach. The foraging model of E.Coli bacteria is used for optimization. The algorithm uses two window sizes; one for zero and one for the rest of the digits. Tested on a small dataset, the average recognition was $96 \%$.

In Ref. [21], during segmentation, the digits are segmented into primitives that are measured and labeled using fuzzy logic. The labeled primitives assist in forming strings of numerals. Conventional string matching along with membership value of strings was used for classification and recognition. In [22], the target is the recognition of Odia handwritten digits using HMM. Feature extraction is done using polygonal approximation of object contour. Fuzzy inference system is then used to generate aggregated feature vectors for each digit. The feature vector for each digit is then split into three levels and used as three different state for HMM. The end result is a HMM model with ten different three state correspond to ten digit classes. Log-likelihood is then used to calculate and decide the class label. The overall accuracy achieved was $96.3 \%$. In [23], a feature selection technique base on Axiomatic Fuzzy Set (AFS) was proposed. 
The technique allows for the most powerful subset of the feature set to be selected based on the class seperability power of combined features. Their method is tested on handwritten numeral in five languages namely Arabic, Telugu, Roman, Devanagari, and Bangla. SVM classifier was used in the study. Higher recognition rates and less features were reported in the study. In [24], the authors presented two hybrid classification techniques for handwritten Arabic numerals. The proposed the serial hybrid classifier consisting of first level Fuzzy C-Means followed by SVM and followed a pixel method classifier. They also proposed the parallel hybrid classifier fusing the decisions of Fuzzy C-Means and Neural Network in parallel to make final decision. Hybrid classifiers were reported to outperform single classifiers. In [25], CNN was combined with a fuzzy logic module based on structural approach. The combination of fuzzy logic was able to increase the recognition rate of handwritten numerals. In [26], Quantum Neural Network (QNN) which combines fuzzy theoretical principles and neural modelling is used for numeral recognition. An average recognition accuracy of $99.1 \%$ was reported.

Due to the noise that has been accumulated from all recognition steps, some numerals will be confused with other numerals in the classifiers output. At this stage, no further processing could be achieved. We propose a method that could be plugged into the output of the classifier that helps in decreasing the confusion among numerals that have close scores at the classifier level. Instead of using binary threshold to make the final decision, we propose a fuzzy threshold using a trapezoidal membership function in the cases where the highest two consecutive scores are close to each other. Particle Swarm Optimization (PSO) were used to minimize the performance index (number of confused numerals).

\section{MATERIALS AND METHODS}

The proposed Methodology and systems consists of four distinct phases; First is the Preprocessing phase, Structural Feature extraction is then achieved in phase 2 , phase 3 consists of the classification, and finally phase 4 is enhancing the classification by processing the misclassified digits using fuzzy logic. The following is the details of the four phases of the proposed system.

\section{A. Preprocessing}

English, Persian, Arabic, and Devanagari digits were specifically chosen for this study due to the structural similarity between digits in these numbers. The representation of the numerals from 0-9 in these languages are shown in Table I.

The challenge in digit recognition is the different handwriting styles. For example, 52 writing classes have been identified for Persian and Arabic numerals [1]. As seen in Table I, some digits are have similarities that make the recognition process more complex. Samples of handwritten numbers in English, Arabic, Persian, and Devanagari are shown in Fig. 1. It is clearly seen from the figure that even though the numerals in these languages were used based on their structural similarity yet this makes the recognition process more difficult because of structural similarities between numbers in the same language or between the different languages.

Preprocessing is a crucial phase in image recognition techniques. It is essential to make the images uniform in terms of size, location, and angle in addition to removing noise from the images. The proposed system images are subject to the following preprocessing steps:

Grayscale to binary conversion through the Otsu's Thresholding Method [27].

- Noise removal using a $3 \times 3$ window of disk shaped structure. Dilation and erosion (morphological operations) are applied for noise removal.

- Boundary box applied to each image for segmentation and separation.

- Centering the segmented numeral based on region centroids.

- Normalization and converting the image to size $36 \times 36$.

TABLE I: Arabic, ENGLish, PERsian and Devanagari Digits

\begin{tabular}{|c|c|c|c|}
\hline English & Arabic (Eastern) & Persian & Devanagari \\
\hline 0 & . & . & 0 \\
\hline 1 & 1 & 1 & $?$ \\
\hline 2 & $r$ & $r$ & ? \\
\hline 3 & $r$ & $r$ & ३ \\
\hline 4 & $\varepsilon$ & p & $\gamma$ \\
\hline 5 & 0 & $\Delta$ & 4 \\
\hline 6 & 7 & 9 & $\xi$ \\
\hline 7 & $\mathrm{~V}$ & $\mathrm{~V}$ & ७ \\
\hline 8 & $\Lambda$ & $\Lambda$ & $c$ \\
\hline 9 & 9 & 9 & $\rho$ \\
\hline
\end{tabular}

\begin{tabular}{|c|c|c|c|c|c|c|c|}
\hline \multicolumn{2}{|c|}{ Arabic } & \multicolumn{2}{|c|}{ English } & \multicolumn{2}{|c|}{ Persian } & \multicolumn{2}{|c|}{ Devanagari } \\
\hline S-1 & & S-1 & $\mathrm{S}-2$ & $\mathrm{~S}-1$ & S-2 & S-1 & $\mathrm{S}-2$ \\
\hline - & $c$ & 0 & 0 & ص & - & 0 & c) \\
\hline 1 & 1 & 1 & 1 & 1 & 1 & ? & 9 \\
\hline ( & 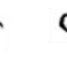 & 2 & 2 & $r$ & 5 & 2 & 2 \\
\hline$r$ & 1 & 3 & 3 & $\kappa$ & $\psi$ & 3 & 3 \\
\hline$\varepsilon$ & - & 4 & 4 & $r$ & $r$ & $\gamma$ & $\gamma$ \\
\hline$c$ & c & 5 & 5 & $x$ & $\diamond$ & $y$ & $y$ \\
\hline 7 & 7 & 6 & 6 & $y$ & $y$ & $\xi$ & $\xi$ \\
\hline$V$ & $v$ & 7 & 7 & $V$ & $\checkmark$ & 6 & 6 \\
\hline 1 & $\wedge$ & 8 & 8 & $\widehat{\Lambda}$ & $\Omega$ & $r$ & $\tau$ \\
\hline . & 。 & 9 & 9 & 4 & 9 & \{ & $\varepsilon$ \\
\hline
\end{tabular}

Fig. 1. Samples of handwritten Arabic, English, Persian and Devanagari numerals.

\section{B. Feature Extraction}

As mentioned in the literature review, many feature extraction methods have been proposed. In our proposed system, we used a structural feature extraction method similar to those proposed in [1]. The feature extraction phase is the most critical phase as it will set the basis of how digits differ from each other in terms of the measurements of the structural features chosen for this method. The similarity between the digits requires that the concentration is on the local structural features rather than on the global features that could be extracted by applying Histogram based algorithms, DFT and DCT. In this work, we propose structural features totaling to 37 features and they extracted based on the 
calculations shown below. The extracted local features are calculated as follows taking into account that the images input to the feature extraction phase are the preprocessed images.

1. In the image, calculations (Horizontally) of the starting black pixel and ending black pixel is obtained over three locations (top, center and bottom) as shown in Fig. 2(a). The same method is used for Horizontal calculation of the starting black pixel and ending black pixel as shown in Fig. 2(b). The total features extracted in this step are equal to 12 features.

2. Another 3 horizontal and 3 vertical features are extracted by measuring the distance between the starting and ending black pixels as shown in Fig. 2(a), Fig. (b) respectively. Equations 1 and 2 are used for the measurement of these features.

$$
\begin{gathered}
H_{-} \text {distance }_{i}=\operatorname{Hor}\left(S_{i}\right)-\operatorname{Hor}\left(E_{i}\right) \\
V_{-} \text {distance }_{i}=\operatorname{Ver}\left(S_{i}\right)-\operatorname{Ver}\left(E_{i}\right)
\end{gathered}
$$

where $H_{-}$distance $i$ and $V_{-}$distance $_{i}$ are the horizontal distance and vertical distance for defined axis vector $i$. $\operatorname{Hor}\left(S_{i}\right)$ and $\operatorname{Hor}\left(E_{i}\right)$ are horizontal first and last appearance of black pixel respectively, and $\operatorname{Ver}\left(S_{i}\right)$ and $\operatorname{Ver}\left(E_{i}\right)$ are vertical first and last appearance of black pixel respectively.

3. The aspect ratio of height to width distance (HW-Aspect) is used also as additional features using equation 3 to calculate the ratio. This gives an extra 3 features for height distance to width distance ratio (H-W_Ratio) and is measured using the horizontal distance and vertical distance as shown in Fig. 2 (c).

$$
H_{-} W_{-} \text {Ratio }_{i}=\frac{\text { Hdist }_{i}}{\text { Wdist }_{i}}
$$

4. Other features are observed by dividing the image into $4 \times$ 4 segments and calculating the number of black pixels in each segment. This adds another 16 features obtained in this step. Fig. 2 (d) shows the segments and Equation 4 shows the equation used for calculating the number of black pixels in each segment.

$$
S_{m}=\sum_{i-1, j=1}^{i=B H, j=B W} S_{m}[i, j]=1
$$

where $S_{m}$ is the sum of the black pixels of segment $m$. $B H$ and $B W$ are segment height and segment width respectively for each block.
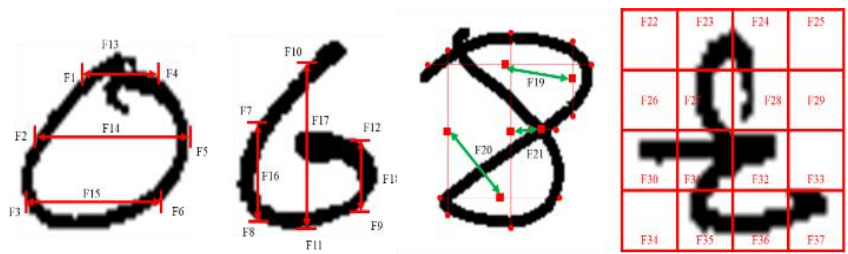

Fig. 2. Graphical representation of Feature extraction methodology. From left to right: (a). Starting and ending $\mathrm{X}$-axis location of the black pixel occurrence and the horizontal distance between them, (b). similarly starting and ending $y$-axis location of the black pixel occurrence and the horizontal distance between them, (c). Height Distance to Width Distance aspect ratio, (d). Features based on the black pixels in 16 blocks.

\section{Classification}

In the classification phase, two classifiers were chosen for the proposed system. The two classifiers are Naïve Bayes and Neural Network. The following a brief about each classifier.

\section{Naïve Bayes}

The basis for Naïve Bayes classifier is the Bayes' theorem for probabilistic statistical model. The conditional probability of assigning a data to a certain class is calculated using both the condition and unconditional probability of the data belonging to each class in the all the data set. The Naïve Bayes classifier has extensively documented in previous literature [28]-[30].

Thus $\mathrm{P}\left(x_{i} \mid c_{k}\right)$ probability (likelihood function) values are calculated as an estimate from the training sample of the Numeral image set with attributes $x_{i}$ which can be represented with the value $s_{i k}$ using equation 5 .

$$
\mathrm{P}\left(x_{i k} \mid c_{k}\right)=\frac{s_{i k}}{n}
$$

The summation training samples for attribute $x_{i k}$ in class $c_{k}$ is specified by $s_{i k}$, while $n$ is the training sample number in class $k$. For simplicity, $\mathrm{P}\left(x_{i} \mid c_{k}\right)$ is modeled as normal density function for each attribute. To find the class $k$ maximum probability, the Naïve Bayes classifier can be used and is specified by equation 6 .

$$
\underset{k}{\operatorname{argmax}} \mathrm{P}\left(c_{k}\right) \prod_{i=1}^{n} \mathrm{P}\left(x_{i} \mid c_{k}\right)
$$

Data is divided into training and testing data in the Naïve Bayes classification dataset. The training dataset is considered as previous information and the model is constructed on the basis of the training set.

\section{Neural Networks (Multilayer Perceptron)}

Multilayer Perceptron (MLP), a basic type of deep learning algorithm, is a classifier containing hidden interconnected layers. MLP has extensively been documented in previous literature [31-33]. Matrices are used in MLP in which a matrix $\mathrm{M}_{\mathrm{i}}$ uses matrix multiplication to encode conversions through layers. The transformation matrix of $x$ neurons in a layer connected to $y$ neuron of the consecutive layer has a dimension shown in equation 7 .

$$
M \epsilon R^{x * y}
$$

The transformation layer has the capability of mapping the input to the output using equation 8,9 , and 10 .

$$
\begin{gathered}
\text { Input } \epsilon R^{x * 1} \\
\text { Output } \epsilon R^{1 * y} \\
\text { output }=\text { Input }^{T} M
\end{gathered}
$$

\section{Fuzzy Set-Based Optimization}

After the classification phase, some digits will still be misclassified or erroneously classified. We therefore introduce this fourth layer of Fuzzy Set-based Optimization which will process the erroneously classified digits and reclassify them reducing the number of erroneously classified digits and increasing the overall accuracy and recognition rate of the proposed system. In this paper, we propose the use of the Trapezoidal Fuzzy sets with the following membership function with parameters $w, x, y$, and $z$ as sown in Equation 11 and Fig. 3.

The trapezoidal fuzzy sets have been used and extensively explained in previous literature [34], [35]. 


$$
A=\left\{\begin{array}{c}
0, m \leq w \\
\frac{m-w}{x-w}, w \leq m \leq x \\
1, x \leq m \leq y \\
\frac{z-m}{z-c}, y \leq m \leq z \\
0, z \leq m
\end{array}\right.
$$

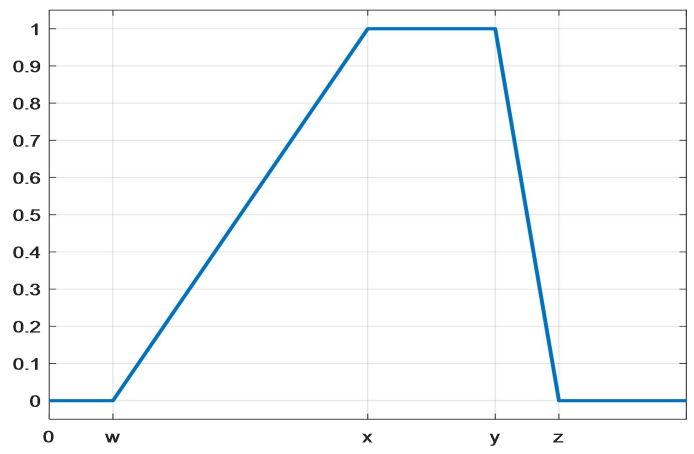

Fig. 3. Trapezoidal membership function.

The parameters of the trapezoidal function are optimized using PSO. PSO is considered an evolutionary optimization technique. The number of parameters of any two confused numerals is 8 two for two membership functions. The objective of the fitness function is to minimize the confusion between the numerals. Let us assume the normalized highest two consecutive scores at the classifier output are 0.51 for class 1 and 0.49 for class 2 and assuming the true ground is class 2. For the purpose of illustration of two-class problem, we subtract 1 from class 2 which result in 0.51 (the same as class 1 score). Then, if the resulted score is greater than 0.5 then the decision will go for class 1 otherwise for class 2 .

The decision in this case will be erroneously made for class 1. While in the proposed approach, we augment this score by two membership function for each class as shown in Fig. 4. The mapped values for the score 0.51 is 0.73 and 0.82 for class 1 and class 2 respectively. Thus, the decision will be correctly made in this way for class 2 . It should be noted that this method will focus on normalized scores (classifier output) that are between 0.45 and 0.55 as widening this range will effect on the correctly classified classes. The formation of the two trapezoidal membership functions between any two classes in the context of numeral recognition problem will be obtained from as mentioned earlier by PSO technique. The parameters of PSO are: Swarm size 100, number of generations 100, cognitive acceleration coefficient, and the social acceleration coefficient were set to 2 .

\section{EXPERIMENTAL DATA}

The type and size of the reference database may affect the results obtained from any proposed technique. The dataset used in the paper are those documented in previous literature and contain a large number of sample which make the results obtained using the proposed model comparable to those published in previous literature. The proposed model is validated with four well-known large databases for the four targeted languages totaling more than 190,000 numeral pattern samples. The first database used is the Modified Arabic Handwritten Digits Databases (MADBase) collected from 700 individuals [36] containing 70,000 handwritten Arabic digits in 300 dpi resolution in $28 \times 28$ pixels. For
English Numerals, the MNIST Dataset was used. Modified National Institute of Standards and Technology (MNIST) dataset consisted of 70,000 samples collected from 250 participants [37]. For Persian, the HODA dataset was used which the first Farsi handwritten digit dataset extracted from 12000 registration forms. It consists of a total 80,000 samples [38]. For the Devanagari numerals, the Devanagari Handwritten Character Dataset (DHCD) was used which consists of a total of 20,000 samples collected from an unspecified number of documents [39].

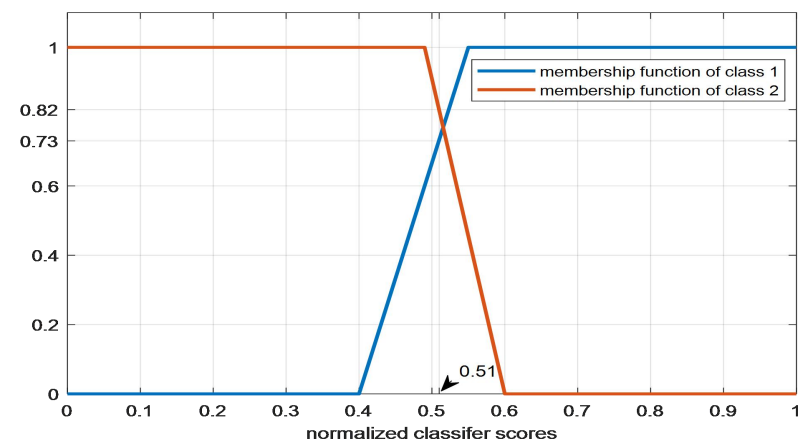

Fig. 4. Quantifying the classifier's scores using trapezoidal membership functions.

TABLE II: NumERALS DATASETS USED FOR PROPOSED SySTEM

\begin{tabular}{|l|l|l|l|l|}
\hline Language & Name & Source & $\begin{array}{l}\text { Training } \\
\text { Numerals }\end{array}$ & $\begin{array}{l}\text { Testing } \\
\text { Numerals }\end{array}$ \\
\hline Arabic & MADBase & $\begin{array}{l}700 \\
\text { volunteers }\end{array}$ & 60,000 & 10,000 \\
\hline English & MNIST & $\begin{array}{l}250 \\
\text { participants }\end{array}$ & 60,000 & 10,000 \\
\hline Persian & HODA & 12000 Forms & 60,000 & 20,000 \\
\hline Devanagari & DHCD & NA & 17,000 & 3,000 \\
\hline
\end{tabular}

\section{RESUlts AND Discussions}

Table III shows the number of digits whose normalized scores are between 0.45 and 0.55 both using Neural Network $(\mathrm{NN})$ and Naïve Bayes (NB) classifiers. It is noticed that the number of digits normalized between these scores are much higher using $\mathrm{NN}$ than those normalized into these scores using NB. For example, in training of Arabic Numerals 50 digits had the normalized score between 0.45 and 0.55 as opposed to only 16 digits using NB. We note here that these represent the total number of digits, meaning both classified correctly and misclassified (erroneously classified) by the system.

Fig. 5 shows the average fitness value of the swam in each generation in one of the confusion cases, it is clearly shown that parameters of membership functions were converged to values in which the number of misclassified numerals is zero for this particular case.

TABLE III: Number OF Numerals WITH NORMALIZED SCORE BETWEEN 0.45 AND 0.55 USING NEURAL NETWORK AND NA VE BASE

\begin{tabular}{|l|l|l|l|l|l|l|}
\hline \multicolumn{1}{|l}{ Total } & \multicolumn{2}{l}{ NN } & \multicolumn{2}{l|}{ NB } \\
\hline \multirow{4}{*}{ Language } & \multirow{4}{*}{ Train } & \multirow{4}{*}{ Test } & $\begin{array}{l}\text { Train } \\
0.45>\end{array}$ & $\begin{array}{l}\text { Test } \\
0.45>\end{array}$ & $\begin{array}{l}\text { Train } \\
0.45>\end{array}$ & $\begin{array}{l}\text { Test } \\
0.45> \\
\end{array}$ \\
& & & NN $>$ & NN $>$ & NB $>$ & NB $>$ \\
& & 0.55 & 0.55 & 0.55 & 0.55 \\
\hline Arabic & 60000 & 10000 & 301 & 50 & 104 & 16 \\
\hline English & 60000 & 10000 & 1001 & 165 & 285 & 49 \\
\hline Persian & 60000 & 20000 & 350 & 190 & 147 & 58 \\
\hline Devanagari & 17000 & 3000 & 265 & 52 & 125 & 22 \\
\hline
\end{tabular}




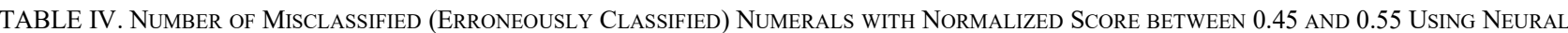
NeTWORK COMPARED WITH THE ADDED FuZZY PHASE AND NA VE BASE COMPARED WITH THE ADDED FuZZy PHASE

\begin{tabular}{|c|c|c|c|c|c|c|c|c|c|c|c|c|}
\hline \multirow[b]{3}{*}{ Language } & \multicolumn{6}{|c|}{ NN } & \multicolumn{6}{|c|}{ NB } \\
\hline & \multicolumn{3}{|c|}{ Training } & \multicolumn{3}{|c|}{ Testing } & \multicolumn{3}{|c|}{ Training } & \multicolumn{3}{|c|}{ Testing } \\
\hline & $\begin{array}{l}\text { No } \\
\text { Fuzzy }\end{array}$ & $\begin{array}{l}\text { Using } \\
\text { Fuzzy }\end{array}$ & $\begin{array}{l}\% \\
*\end{array}$ & $\begin{array}{l}\text { No } \\
\text { Fuzzy }\end{array}$ & $\begin{array}{l}\text { Using } \\
\text { Fuzzy }\end{array}$ & $\begin{array}{l}\% \\
*\end{array}$ & $\begin{array}{l}\text { No } \\
\text { Fuzzy }\end{array}$ & $\begin{array}{l}\text { Using } \\
\text { Fuzzy }\end{array}$ & $\begin{array}{l}\% \\
*\end{array}$ & $\begin{array}{l}\text { No } \\
\text { Fuzzy }\end{array}$ & $\begin{array}{l}\text { Using } \\
\text { Fuzzy }\end{array}$ & $\begin{array}{l}\% \\
*\end{array}$ \\
\hline Arabic & 139 & 81 & 41.73 & 26 & 21 & 19.23 & 51 & 19 & 62.75 & 8 & 3 & 62.50 \\
\hline English & 436 & 332 & 23.85 & 84 & 69 & 17.86 & 157 & 83 & 47.13 & 26 & 22 & 15.38 \\
\hline Persian & 175 & 122 & 30.29 & 96 & 86 & 10.42 & 70 & 29 & $\begin{array}{l}58.57 \\
\end{array}$ & 32 & 22 & 31.25 \\
\hline Devanagari & 127 & 83 & 34.65 & 29 & 26 & 10.34 & 60 & 24 & 60.00 & 12 & 9 & 25.00 \\
\hline
\end{tabular}

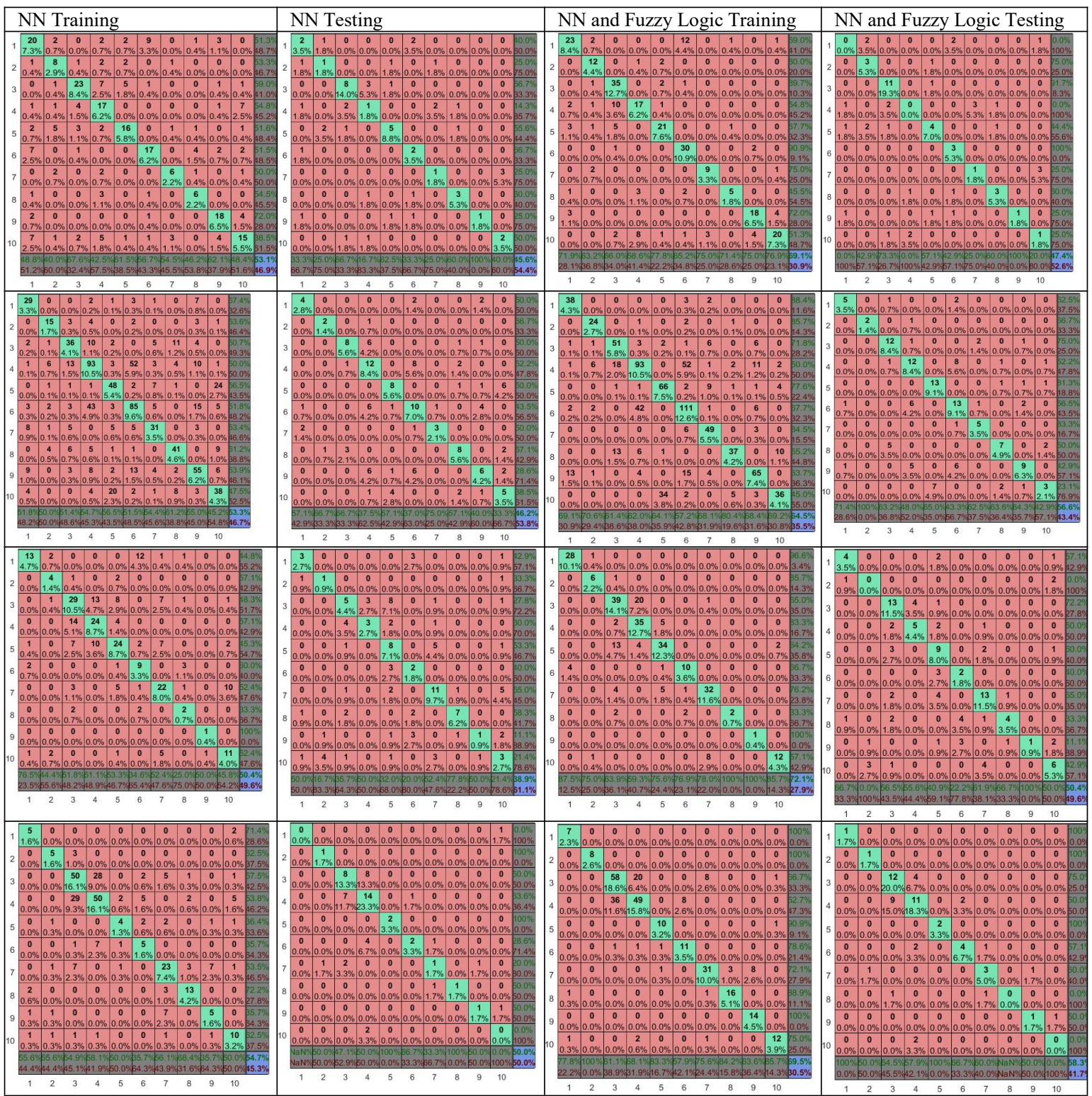

Fig. 5. Confusion matrixes based on the output class (Horizontal) verses target class (Vertical) for 10 different possible numerals. Each row from top to bottom represent results for Arabic, English, Persian and Devanagari using fuzzy based neural networks classifier

Table IV shows the number of misclassified (erroneously classified) digits both with the Fuzzy Phase and without the fuzzy phase (Classifiers only). It is evident in this table that the number of misclassified digits is reduced in all cases and in all datasets using the optimized Trapezoidal Fuzzy sets.

The percentage of this decrease is also shown for both training and testing. This is results in an overall average recognition accuracy.

Fig. 7 shows the confusion matrix using the $\mathrm{NN}$ alone and using the NN followed by the Trapezoidal Fuzzy sets phase. It is shown in these tables that the number of confused digits is reduced when using the $\mathrm{NN}$ with the Trapezoidal Fuzzy sets Phase.

Fig. 6 shows the confusion matrix using the NB alone and using the NB followed by the Trapezoidal Fuzzy sets phase. It is shown in these tables that the number of confused digits is reduced when using the Naïve Base with the Trapezoidal Fuzzy sets Phase.

This method is aimed to improve the classification rate in handwritten numeral recognition problem regardless the classifier's type. Therefore, comparative analysis in this case is meaningless because improvement is achieved after obtaining the result of the classifier by hooking the fuzzy based method up with the classifier output. 


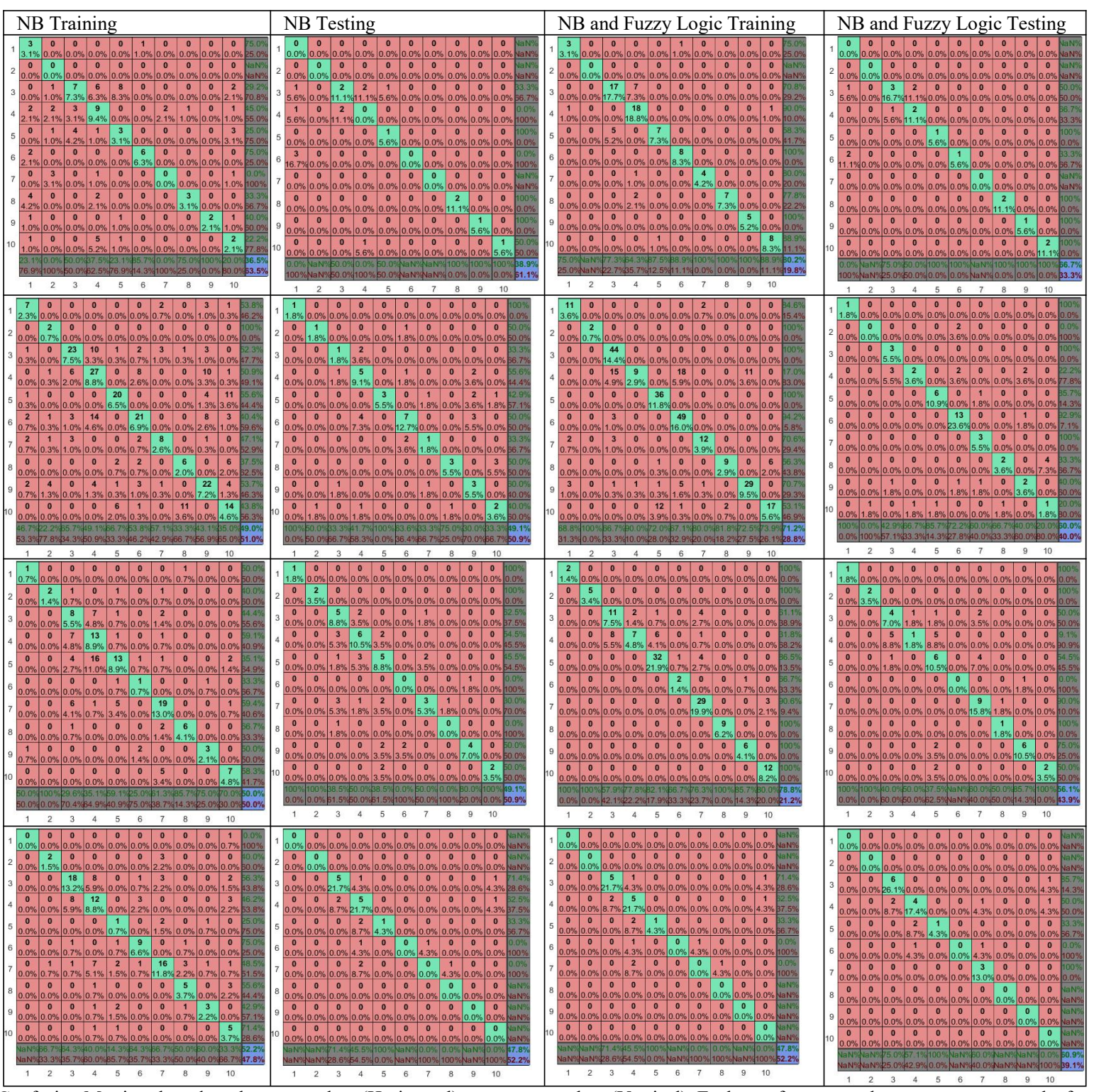

Fig. 6. Confusion Matrixes based on the output class (Horizontal) verses target class (Vertical). Each row from top to bottom represent results for Arabic, English, Persian and Devanagari using Fuzzy based Naïve Bayes classifier.

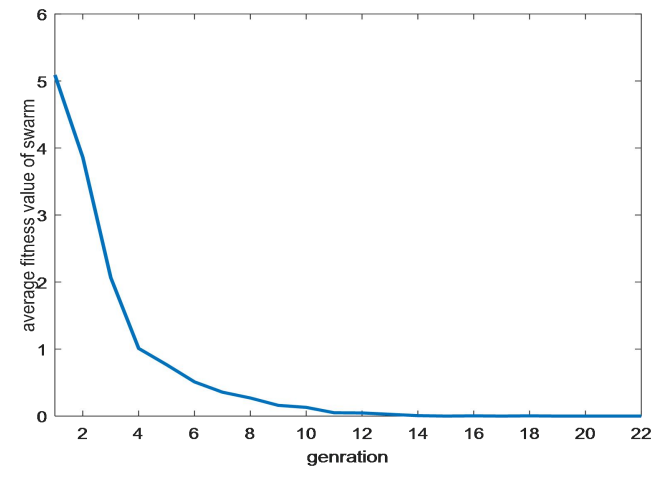

Fig. 7. Values of average fitness function of swam in successive generations.

\section{CONCLUSION}

In this paper we propose a four phase recognition system with the fourth phase being the Fuzzy Based Decision (Trapezoidal Fuzzy sets) rather than the usual three phase recognition systems which constitute of Pre-Processing, Feature Extraction and Classification. The Preprocessing phase consists of steps to normalize the images and denoised them as well centralization of the handwritten numeral. The feature extraction phase is based on the structural features of the handwritten numerals written in the four languages targeted in this paper namely; English, Arabic, Persian and Devanagari. For Classification, two well-known classifiers were chosen namely; MLP and Naïve Bayes classifier. The fourth phase consists of the use of Trapezoidal Fuzzy sets with the membership functions specified in the body of the paper. It is clearly evident from the results produced that the number of misclassified digits is further reduced by using the Fuzzy Set-Based Decision Mechanism which increase the overall average recognition accuracy of the system.

Future work will include designing a numeral recognition system language independent, writer independent using deep learning networks combined with Fuzzy Set-Based Decision Mechanism.

\section{CONFLICT OF INTEREST}

The authors declare no conflict of interest.

\section{AUTHOR CONTRIBUTIONS}

A. Al-Hmouz, G. Latif, J. Alghazo conducted the research; A. Al-Hmouz, R. Al-Hmouz analyzed the data; All authors 
wrote the paper; All authors had wrote the paper and approved the final version.

\section{REFERENCES}

[1] J. M. Alghazo, G. Latif, A. Elhassan, L. Alzubaidi, A. Al-Hmouz, and R. Al-Hmouz, "An online numeral recognition system using improved structural features- a unified method for handwritten arabic and persian numerals," Journal of Telecommunication, Electronic and Computer Engineering (JTEC), vol. 9, no. 2-10, pp. 33-40, 2017.

[2] M. A. Abuzaraida, A. M. Zeki, and A. M. Zeki, "Online recognition system for handwritten Arabic digits," in Proc. the 7th International Conference on Information Technology, Amman, Jordan, 2015, pp. 45-49.

[3] R. Al-Hmouz, "OCR Based Pixel Fusion," Journal of Applied Sciences, vol. 12, pp. 2319-2325, 2012.

[4] S. A. Azeem, M. El Meseery, and H. Ahmed, "Online Arabic handwritten digits recognition," in Proc. International Conference on Frontiers in Handwriting Recognition (ICFHR), 2012, pp. 135-140.

[5] A. G. Ramakrishnan and K. B. Urala, "Global and local features for recognition of online handwritten numerals and Tamil characters," in Proc. the 4th International Workshop on Multilingual OCR, 2013, pp. 16-21.

[6] V. Ghods and M. K. Sohrabi, "Online farsi handwritten character recognition using hidden markov model," $J C P$, vol. 11 , no. 2, pp. 169-175, 2016.

[7] B. E. Kessab, C. Daoui, B. Boukhalene, and R. Salouan, "A comparative study between the k-nearest neighbors and the multi-layer perceptron for cursive handwritten arabic numerals recognition," International Journal of Computer Applications, vol. 107, no. 21, pp. 25-30, 2014.

[8] R. Salouan, S. Safi, and B. Bouikhalene, "Printed eastern arabic noisy numerals recognition using hidden markov model and support vectors machine," International Journal of Innovation and Applied Studies, vol. 9, no. 3, pp. 1032-1042, 2014.

[9] R. Azad, F. Davami, and H. S. Boroujeni, "Recognition of handwritten Persian/Arabic numerals based on robust feature set and K-NN classifier," International Journal of Computer and Information Technologies, vol. 1, no. 3, pp. 220-230, 2013.

[10] S. Khorashadizadeh and A. Latif, "Arabic/Farsi handwritten digit recognition using histogram of oriented gradient and chain code histogram," International Arab Journal of Information Technology (IAJIT), vol. 13, no. 4, pp. 1-8, 2016.

[11] H. A. Alwzwazy, H. M. Albehadili, Y. S. Alwan, and N. E. Islam, "Handwritten digit recognition using convolutional neural networks," International Journal of Innovative Research in Computer and Communication Engineering, vol. 4, no. 2, pp. 1101-1106, 2016.

[12] H. Karimi, A. Esfahanimehr, M. Mosleh, S. Salehpour, and O. Medhati, "Persian handwritten digit recognition using ensemble classifiers," Procedia Computer Science, vol. 73, pp. 416-425, 2015.

[13] A. Boukharouba and A. Bennia, "Novel feature extraction technique for the recognition of handwritten digits," Applied Computing and Informatics, vol. 13, no. 1, pp. 19-26, 2015.

[14] A. El-Sawy, E. B. Hazem, and M. Loey, "CNN for handwritten Arabic digits recognition based on LeNet-5," in Proc. International Conference on Advanced Intelligent Systems and Informatics, pp. 566-575, 2016.

[15] G. Latif, J. Alghazo, L. Alzubaidi, M. M. Naseer, and Y. Alghazo, "Deep convolutional network for recognition of unified multi-language handwritten numerals," in Proc. 2nd IEEE International Workshop on Arabic and Derived Script Analysis and Recognition (ASAR), March 2018.

[16] D. Musleh, K. Halawani, and S. Mahmoud, "Fuzzy modeling for handwritten arabic numeral recognition," International Arab Journal of Information Technology (IAJIT), vol. 14, no. 4, pp. 1-10, 2015.

[17] M. Hanmandlu and O. V. R. Murthy, "Fuzzy model based recognition of handwritten numerals," Pattern Recognition, vol. 40, no. 6, pp. 1840-1854, June, 2007.

[18] C. Jou and H. C. Lee, "Handwritten numeral recognition based on simplified structural classification and fuzzy memberships," Expert Systems with Applications, vol. 36, no. 9, 2009, pp. 11858-11863, 2009.

[19] O. V. R. Murthy and M. Hanmandlu, "Interactive fuzzy model based recognition of handwritten characters," Journal of Pattern Recognition Research, vol. 2, pp. 154-165, May 2011.

[20] M. Hanmandlu, A. V. Nath, A. C. Mishra, and A. C. Madasu, "Fuzzy model based recognition of handwritten hindi numerals using bacterial foraging," in Proc. Int. Conf. Comput. Inf. Sci., 2007, pp. 309-314.

[21] R. M. Suresh and S. Arumugam, "Fuzzy technique based recognition of handwritten characters," Image and Vision Computing, vol. 25, no. 2, pp. 230-239, 2007

[22] T. K. Mishra, B. Majhi, P. K. Sa, and S. Panda, "Model based odia numeral recognition using fuzzy aggregated features," Frontiers of Computer Science, vol. 8, no. 6, pp. 916-922, 2014.

[23] A. Roy, N. Das, R. Sarkar, S. Basu, M. Kundu, and M. Nasipuri, “An Axiomatic Fuzzy Set Theory Based Feature Selection Methodology for Handwritten Numeral Recognition," in Proc. 48 ${ }^{\text {th }}$ Annual Convention of Computer Society of India, vol. 1, AISC248, pp. 133-140, 2014.

[24] M. Takruri, R. Al-Hamouz, A. Al-Hamouz, and M. Momani, "Fuzzy C-Means based hybrid classifiers for offline recognition of handwritten Indian (Arabic) numerals," International Journal of Applied Engineering Research, vol. 10, no. 11, pp. 1911-1924, 2015.

[25] E. A. Popko and I. A. Weinstein, "Fuzzy logic module of convolutional neural network for handwritten digits recognition," Journal of Physics: Conference Series, vol. 738, no. 1, 2016.

[26] J. Zhou, "Recognition of handwritten numerals by quantum neural network with fuzzy feature," Int. J. Document Anal. Recognit., vol. 2, no. 1, pp. 30-36, 1999.

[27] J. L. Fan and F. Zhao, "Two-dimensional Otsu's curve thresholding segmentation method for gray-Level images," Dianzi Xuebao(Acta Electronica Sinica), vol. 35, no. 4, pp. 751-755, 2007.

[28] P. Cichosz, "Naïve Bayes classifier," Data Mining Algorithms: Explained Using, 2015.

[29] D. Berend and A. Kontorovich, "A finite sample analysis of the Naive Bayes classifier," J. Mach. Learn. Res.. vol. 16, pp. 1519-1545, 2015.

[30] H. J. Escalante, E. F. Morales, and L. E. Sucar, "A na"1ve bayes baseline for early gesture recognition," $P R L$, vol. 73, pp. 91-99, 2016.

[31] S. K. Pal and S. Mitra, "Multilayer perception, fuzzy sets and classification," IEEE Trans Neural Netw., vol. 3, no. 5, pp. 683-696, 1992.

[32] S. Haykin, Neural Networks \& Learning Machines, Prentice-Hall, 2008.

[33] H. Cartwright, Artificial Neural Networks, Humana Press, 2015.

[34] R. Al-Hmouz and W. Pedrycz, "Models of time series with time granulation," Knowledge \& Information Systems, vol. 48, no. 3, pp. 1-20, 2016.

[35] Z. Xiao, S. Xia, K. Gong, and D. Li, "The trapezoidal fuzzy soft set and its application in MCDM," Applied Mathematical Modelling: Simulation and Computation for Engineering and Environmental Systems, vol. 36, no. 12, pp. 5844-5855, 2012.

[36] S. Abdelazeem, "Comparing arabic and latin handwritten digits recognition problems," World Academy of Science, Engineering and Technology, vol. 3, no. 6, pp. 1583-1587, 2009.

[37] Y. LeCun, L. Bottou, Y. Bengio, and P. Haffner, "Gradient-based learning applied to document recognition," in Proc. the IEEE, vol. 86, no. 11, pp. 2278-2324, 1998.

[38] H. Khosravi and E. Kabir, "Introducing a very large dataset of handwritten Farsi digits and a study on their varieties," Pattern Recognit Lett, vol. 28, no. 10, pp. 1133-1141, 2007.

[39] S. Acharya, A. K. Pant, and P. K. Gyawali, "Deep learning based large scale handwritten Devanagari character recognition," in Proc. 2015 9th International Conference on Software, Knowledge, Information Management and Applications (SKIMA), 2015, pp. 1-6.

Copyright (C) 2020 by the authors. This is an open access article distributed under the Creative Commons Attribution License which permits unrestricted use, distribution, and reproduction in any medium, provided the original work is properly cited (CC BY 4.0).

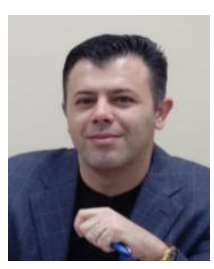

numerals.

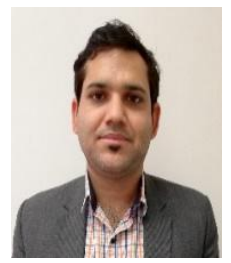

Ahmed Al-Hmouz received the BSc degree in computer science from Mutah University, Jordan. He received the MSc degree in internet technology, the $\mathrm{MSc}$ degree in information systems, and the $\mathrm{PhD}$ degree in the School of Information Systems \& Technology, Faculty of Informatics at the University of Wollongong, Australia. His primary research interests include machine learning, fuzzy decision, and Arabic

Ghazanfar Latif is a PhD scholar at the University of Malaysia Sarawak, Malaysia. He earned his MS degree in computer science from King Fahd University of Petroleum and Minerals, Saudi Arabia in 2014 and BS degree in computer science from FAST National University of Computer and Emerging Sciences, Pakistan in 2010 by remaining in Dean's honor list. Throughout his educational carrier, he got a number of 
achievements like full scholarship for FSc, BS -CS and MS-CS. He worked as an instructor at Prince Mohammad bin Fahd University, Saudi Arabia for 3 years in CS Department and have 2 years industry work experience. His research interests include image processing, artificial intelligence, neural networks, and medical image processing.

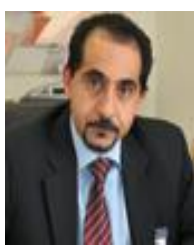

Jaafar Al. Ghazo obtained his $\mathrm{PhD}$ and $\mathrm{MSc}$ in computer engineering from Southern Illinois University Carbondale in 2004 and 2000 respectively. He joined Prince Mohammad Bin Fahd University (PMU) as a founding dean of the College of Computer Engineering and Science and held various positions including dean of Graduate Studies and Research, Dean of Institutional Relations, and dean of Continuing Education and Community Service. Currently he is Assistant Professor at PMU. His research interests include, modelling and realization of biological mechanism using CAD and FPGAs, modelling and realization of arithmetic operations using CAD and FPGAs, low power cache design, and assistive technology for students with disabilities.

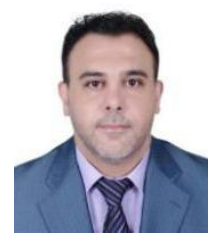

Rami Al-Hmouz received the BSc degree in electrical engineering / telecommunication from Mutah University in 1998, the MSc degree in electrical engineering/communication from the University of Jordan in 2002, the MSc degree in computer engineering from the University of Western Sydney in 2004, and the PhD degree in computer engineering from the University of Technology, Sydney, in 2008. Currently, he is an associate professor at King Abdulaziz University in Saudi Arabia. 